\title{
Experimental indication for supercurrents carried by opened transport channels
}

\author{
L. C. Mur, ${ }^{*}$ C. J. P. M. Harmans, and J. E. Mooij \\ Department of Applied Physics and Delft Institute of Micro-electronics and Submicron-technology (DIMES), \\ Delft University of Technology, P.O. Box 5046, 2600 GA Delft, The Netherlands \\ J. F. Carlin, A. Rudra, and M. Ilegems \\ Institute for Micro- and Optoelectronics, Department of Physics, Ecole Polytechnique Fédérale de Lausanne, \\ CH-1015 Lausanne, Switzerland
}

(Received 2 February 1996)

\begin{abstract}
Electrical-transport properties of mesoscopic junctions consisting of a ballistic two-dimensional electron gas coupled at two sides to overlaying superconducting contacts are presented. The properties suggest that, despite the presence of transport limiting barriers in the sample, supercurrents are carried by a few modes that have near-unity transmission probability. We can qualitatively account for the presence of these resonances by the theory of disorder-induced opening of tunnel channels. [S0163-1829(96)51028-3]
\end{abstract}

Over the past few years, transport properties of phasecoherent normal $(N)$ conductors coupled to superconducting $(S)$ electrodes have received considerable interest. In such structures the interaction between the superconducting condensate and a normal electron gas can be studied on a mesoscopic scale. The process responsible for this interaction is Andreev reflection: an electron incident from $N$ on a $N-S$ interface can be backscattered as a hole, which has the phase of the incident electron shifted by an amount depending on energy and the macroscopic phase of $S$. As a result of this scattering process transport properties of such hybrid structures can change drastically. ${ }^{1}$ We focus on transport properties of InAs-based superconductor two-dimensional electron gas superconductor junctions $(S-2 \mathrm{DEG}-S)$, where the $2 \mathrm{DEG}$ is in the ballistic regime. Several theoretical ${ }^{2-4}$ and experimental papers ${ }^{5-7}$ describing these devices have been published. However, presently there is no agreement between the predicted and observed value of the critical current $I_{c}$ nor of its temperature dependence $I_{c}(T)$.

In this paper, data on the finite bias differential resistance, $I_{c}$ and $I_{c}(T)$ of $S$-2DEG-S junctions will be discussed in detail and compared to theoretical predictions. It will become clear that the data indicate that, despite the presence of transport limiting regions in the device, $I_{c}$ is carried by a few resonant modes. The presence of these modes with nearunity transmission probability can be qualitatively accounted for by the theory of disorder-induced opening of tunnel channels. ${ }^{8}$ The applicability of this theory will be discussed. We will also show that extensive filtering at low temperature is a prerequisite to obtain the intrinsic transport properties of our junctions.

The basis of the devices is a high-mobility 2DEG confined to a 10 -nm-thick strained $\operatorname{In}_{0.8} \mathrm{Ga}_{0.2} \mathrm{As}$ quantum well sandwiched between a 60-nm InP cap layer and a 300-nm InP buffer layer grown on a SI-InP substrate by chemical beam epitaxy. ${ }^{9}$ The fabrication process can be separated in two steps. ${ }^{10}$ First, the quantum well is locally exposed by selective wet etching. The exposed areas are pairs of parallel rectangles of dimensions $W \times 500 \mathrm{~nm}(W=0.5,1$, or $4 \mu \mathrm{m})$ spaced by $500 \mathrm{~nm}$. Because the etching is anisotropic the actual junction length $L$ is approximately $600 \mathrm{~nm}$. The top inset of Fig. 1 shows a schematic cross section of the left contact. In the same fabrication step a trench is etched to within $100 \mathrm{~nm}$ of the rectangles to limit parallel conduction. In the second step the $\mathrm{Nb}$ electrodes are defined; these electrodes cover the locally exposed rectangular regions of the $\mathrm{In}_{0.8} \mathrm{Ga}_{0.2}$ As quantum well and widen to bonding pads. Prior to $\mathrm{Nb}$ evaporation surface contaminants and oxides are removed by Ar-ion cleaning under vacuum condition. Metal contacts to $\operatorname{In}_{x} \mathrm{Ga}_{1-x}$ As with $x \geqslant 0.8$ have no Schottky barrier, ${ }^{11}$ which in combination with ion cleaning leads to transparent interfaces. Magnée et al. ${ }^{12}$ have shown that $\mathrm{Ar}$ etching of an InAs quantum well increases the electron density while at the same time decreasing the mobility (note that

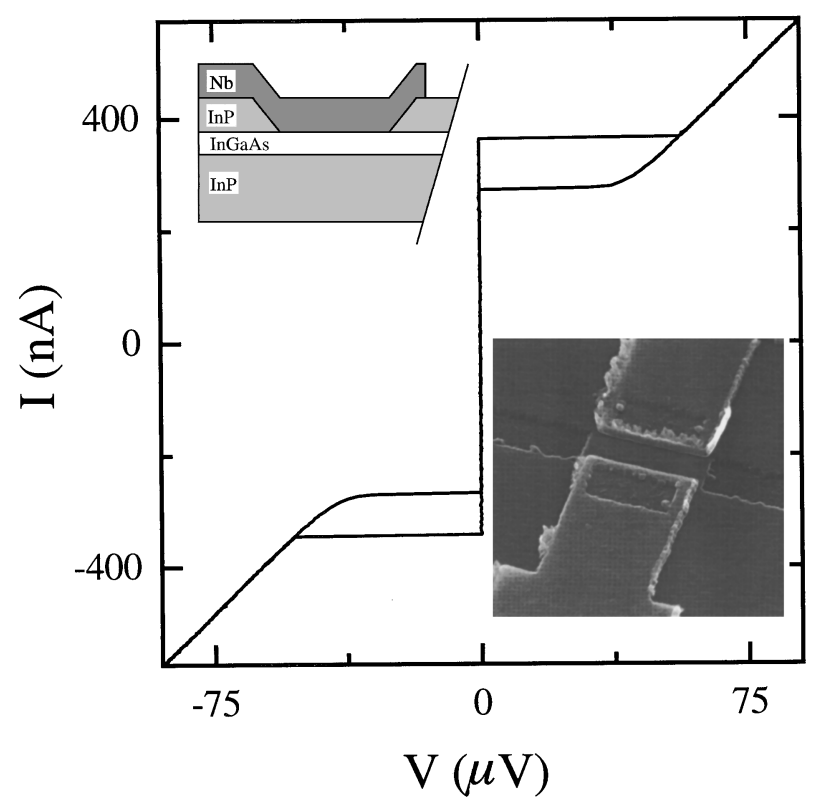

FIG. $1 . I-V$ curve of the $W=4 \mu \mathrm{m}$ junction measured at $10 \mathrm{mK}$ with cryogenic filtering. Top inset: schematic cross section the left $S$-2DEG interface region. Bottom inset: SEM micrograph of a $1-\mu \mathrm{m}$-wide junction. The light areas are the two $\mathrm{Nb}$ electrodes. 
this etching does not affect the 2DEG between the electrodes). The combination of Ar etching and the deposition of the $\mathrm{Nb}$ on top of the quantum well is characteristic of the fabrication process of InAs-based junctions described in literature. The bottom inset of Fig. 1 shows a scanning electron microscope (SEM) micrograph of a completed sample.

By illuminating the 2DEG the electron density, $n_{s}$ and mobility $\mu$ can be set between $3.3-7.0 \times 10^{15} \mathrm{~m}^{-2}$ and $5.0-12 \mathrm{~m}^{2} \mathrm{~V}^{-1} \mathrm{~s}^{-1}$, respectively. Because the resulting mean free path $(0.5 \leqslant l \leqslant 1.7 \mu \mathrm{m})$ is longer than $L$, transport between the electrodes is ballistic. The superconducting coherence length $\xi_{0}=\hbar v_{F} / \pi \Delta \leqslant 70 \mathrm{~nm}$ is shorter than $L$ and so the samples are in the long junction regime. $\Delta$ is the superconducting gap of $\mathrm{Nb}$ and $v_{F}$ the Fermi velocity in the 2DEG, which is calculated by estimating the effective electron mass to be 0.05 times the free-electron mass.

Measurements are performed in a dilution refrigerator with a base temperature of $10 \mathrm{mK}$ and strongly filtered leads. To evaluate the quality of the $S$-2DEG contacts the differential resistance $R_{n}$, measured at a bias voltage above $2 \Delta / e$, can be compared to the theoretical minimum resistance $R_{\min }$, which is approximated by the sum of the 2DEG resistance and the Sharvin resistance. Both the 1- and 4$\mu \mathrm{m}$ junction have a resistance of about three times $R_{\min }$, while the $0.5-\mu \mathrm{m}$ junction has a resistance of $5.5 R_{\min }$. This implies that the average transmission probability per mode is close to $\frac{1}{3}$. When decreasing the bias voltage below $2 \Delta / e$ the differential resistance of all samples dropped which indicates a high tunnel probability $\Gamma$ at the interfaces $;{ }^{13}$ we find $\Gamma \approx 0.8$. Assuming that both $S$-2DEG interface regions can be described by a tunnel barrier and an Ar-etching-induced diffusive region with a transmission probability $T_{d}$ in series, a simple estimate for $T_{d}$ can be made. For this we use the following recursive formula:

$$
T_{N}=\frac{T_{N-1} T^{N}}{1 \pm\left(1-T_{N-1}\right)\left(1-T^{N}\right)},
$$

where a superscript $N$ denotes the transmission probability of the $N$ th barrier and a subscript $N$ the combined transmission probability of a series of $N$ barriers. The plus sign is for phase-coherent transport averaged over phase ${ }^{14}$ and the minus sign for classical transport. Substituting $\Gamma=0.8$ for both interfaces and $T_{4}=1 / 3$ we find $T_{d}=0.8$ and 0.6 for transport in the coherent and incoherent regime, respectively. Consequently, the resistance is just dominated by the diffusive regions. $T_{d}$ might be correlated with $\Gamma$ since a decrease of $\Gamma$ will increase the distance traveled in the disordered region below the superconductor and so decrease $T_{d}$.

Figure 1 shows the current-voltage characteristic $(I-V)$ of the $W=4 \mu \mathrm{m}$ junction at $10 \mathrm{mK}$, which has a current density of almost $100 \mathrm{nA} / \mu \mathrm{m}$. For ballistic Nb-2DEG-Nb junctions of similar electron density, junction length, and effective mass, Chrestin et al. ${ }^{4}$ calculated a critical current density of approximately 20 times the experimental value. Even after taking into account the small differences between the experimental and theoretical device parameters, there still remains an order-of-magnitude difference between theory and experiment. ${ }^{16}$

The critical currents of the $0.5-$ and $1-\mu \mathrm{m}$ junctions are only approximately $1 / 3$ and $2 / 3$ of the values expected from

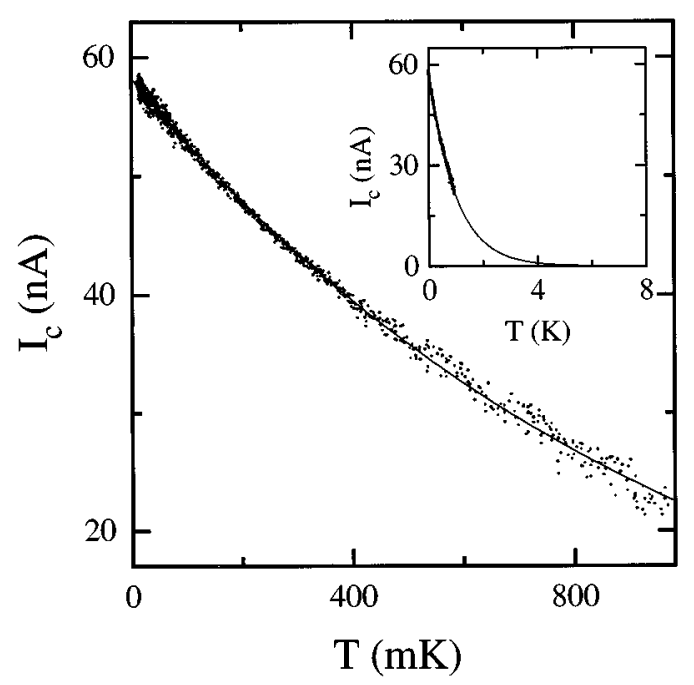

FIG. 2. Critical current vs temperature of the $W=1 \mu \mathrm{m}$ junction measured with cryogenic filtering. The solid line is a $I_{c}(0) \exp \left(-T / T_{0}\right)$ fit to the data. The inset shows the same data and an extrapolation of the fitted temperature dependence on the scale of the critical temperature of $\mathrm{Nb}$.

scaling $I_{c}$ of the $4-\mu \mathrm{m}$ junction. This is probably partly due to the increasing influence of the 100-nm-wide gaps between the mesa and the superconducting contacts. These allow quasiparticles to escape from the junction area, which affects $I_{c}$ more strongly than $R_{n}$.

Figure 2 shows the temperature dependence of the critical current of the $1-\mu \mathrm{m}$-wide junction between $10 \mathrm{mK}$ and $1 \mathrm{~K}$. Each data point represents a separate measurement obtained by automatically recording the value of the bias current at which a voltage develops over the junction. The solid line is a fit to the data: $I_{c}(T)=I_{c}(0) \exp \left(-T / T_{0}\right)$. As can be seen, the fit is quite accurate over a temperature range of two decades. All measurements of $I_{c}(T)$ showed this temperature dependence altough $T_{0}$ varied depending on $W, n_{s}$, and $\mu$.

Two temperature regimes can be distinguished by comparing $L$ to the thermal coherence length $\xi_{T}=\hbar v_{F} / 2 \pi k_{B} T$. In the high-temperature limit, $\xi_{T}<L, I_{c}(T)$ is predicted ${ }^{2,4}$ to be proportional to $\exp \left(-L / \xi_{T}\right)$. This limit is experimentally obtained when $T>1 \mathrm{~K}$. However, thermal rounding of the $I-V$ curves and the smallness of $I_{c}$ in this regime hamper its accurate determination (wider junctions make this regime more accessible). We will concentrate on the lowtemperature regime, $\xi_{T}>L$. In this limit the exponential temperature dependence persists from $1 \mathrm{~K}$ down to the lowest temperature. This is in clear contradiction to the predicted saturation of $I_{c}(T)$ as $T$ approaches zero, ${ }^{2-4,17}$ which occurs whenever quasiparticles have a finite probability of normal reflection at the interfaces. Normal reflection at $S$-2DEG interfaces cannot be completely avoided since there will always be some residual scattering caused by the discontinuity of the conduction bands and the transition between 3D and 2D transport.

The effect of this residual normal scattering on the lowtemperature critical current behavior is quite striking, as can be seen when it is compared to the calculated $I_{c}(T)$ for an 


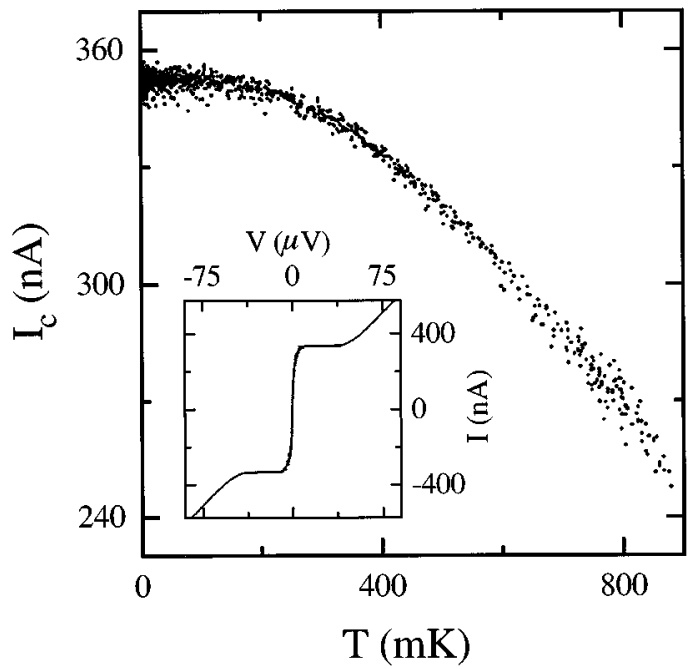

FIG. 3. Critical current vs temperature of the $W=4 \mu \mathrm{m}$ sample measured without cryogenic filters. The inset shows the distorted $I-V$ curve at $10 \mathrm{mK}$.

ideal junction, i.e., only Andreev reflection at the interfaces. For long ballistic junctions $I_{c}$ has the temperature dependence ${ }^{18} I_{c}(T) \propto \exp \left(-L / \xi_{T}\right)$, which qualitatively reproduces the experimental $\exp \left(-T / T_{0}\right)$ dependence. A quantitative comparison can be made when $L$ and $v_{F}$ are substituted. The agreement between theory and the data of the $1-\mu \mathrm{m}$ junction presented in Fig. 2 is within a few percent. Reversing the argument we can obtain an estimate for the effective junction length $L^{*}$ from the experimentally determined parameters $\alpha$ and $v_{F}$. This yields $L^{*} \approx 420 \mathrm{~nm}$ for the $4-\mu \mathrm{m}$ junction, while for the $0.5-\mu \mathrm{m}$ junction $L^{*} \approx 1200 \mathrm{~nm}$ independent of $n_{s}$ and $\mu$. Taking into account the uncertainties in the experimental values of $T_{0}$ and $v_{F}$ and the fact that the actual junction differs considerably from the theoretical model, the quantitative agreement between the ideal junction prediction and the experimental temperature dependence is surprisingly good. The $I_{c}(T)$ characteristic presented by Takayanagi $^{7}$ of a similar ballistic $S$-2DEG- $S$ junction also shows an $\exp \left(-L / \xi_{T}\right)$ temperature dependence in the $\xi_{T}>L$ regime.

The $\exp (-T)$ instead of an $\exp (-\sqrt{T})$ dependence indicates that the sample remains in the clean limit regime ${ }^{2}$ even though at low temperatures $\xi_{T} \gg l, L$. It also shows that phase-breaking effects in the ballistic 2DEG between the electrodes dominate over phase-breaking contributions from transport in the disordered regions underneath the superconductors. This agrees with results from a classical simulation ${ }^{10}$ and experimental data obtained by Magnée et al. ${ }^{15}$ on a similar system that predict quasiparticles will only penetrate approximately $50 \mathrm{~nm}(\ll L)$ into the quantum well underneath the superconductors before being Andreev reflected.

The predictions for $I_{c}(T)$ of junctions with a normal metal or a semiconductor intermediate region can be summarized as follows: ${ }^{17}$ an ideal, fully transparant junction will show an $I_{c}(T)$ curve resembling an exponential behavior (see inset of Fig. 2), whereas a nonideal, partially reflective junction shows a saturation for $T \rightarrow 0$. This leads to the puzzling conclusion that the experimental $I_{c}(T)$ has the depen- dence of an ideal junction, which, however, seems to contradict the facts that $R_{n}$ is three times the minimum resistance and that $I_{c}$ is one order of magnitude smaller than predicted.

We will now briefly discuss the theory of disorder induced opening of tunnel channels, ${ }^{8}$ which can qualitatively explain this surprising result. Even though this theory was derived for particles at the Fermi level while in our junctions transport takes place in a finite energy window, we expect similar effects to take place. The transmission eigenvalues $T_{n}$ of a system containing only a single tunnel barrier are grouped around its transmission probability $T_{b}$. The basic idea of the theory of opened tunnel channels is that if additional scattering is introduced, so that electrons may scatter repeatedly against this barrier, quantum interference can create a few transport channels with near-unity transmission probability $T_{n} \approx 1>T_{b}$. Such multiple scattering against a barrier can be induced by a disordered region, by a second barrier, or by the repeated scattering between the bottom of the quantum well and the superconductor. ${ }^{5}$ In our junctions all three processes may take place. This repeated coherent scattering changes the unimodal distribution of $T_{n}$ to a bimodal one, characterized by a large peak near 0 , the closed channels, and a smaller peak near 1 , the open channels. Note that each $T_{n}$ is associated with a particular superposition of single-mode eigenfunctions. In general these will be diagonal elements of the scattering matrix.

The normal-state conductance does not show critical behavior when these resonant channels appear. ${ }^{8}$ However, the conductance of a $S-N$ junction is more sensitive to the distribution of eigenvalues since closed channels contribute to the conductance with $T_{n}^{2} \ll T_{n}$, while open channels contribute linearly. ${ }^{19}$ An experimental fingerprint of these opened tunnel channels is a peak in the conductance of a $S$-disordered semiconductor junction at small bias voltages and small magnetic fields. This effect was first observed by Kastalsky ${ }^{20}$ while van Wees ${ }^{21}$ identified the interfering trajectories from which the effect originates.

The supercurrent through a long $S$-2DEG-S junction is at least as sensitive to $T_{n}$. This implies that the supercurrent transported by the few open transport channels will strongly dominate over the supercurrent carried by the many closed channels. This allows a qualitative explanation of our data: the zero temperature supercurrent is small since the supercurrent is carried by only a few resonant channels, while it has the temperature dependence of an ideal junction since these channels have near-unity transmission probability.

For simple systems the conditions that have to be met before opened tunnel channels can develop have been analyzed. ${ }^{8}$ In a combined barrier-diffusive-region system the resistance of the diffusive region has to be comparable to or larger than the resistance of the tunnel barrier, while in a double-barrier system open channels develop for approximately equal transmission probabilities of the two barriers. Because, to our knowledge, there is at present no theory available for $S$-2DEG-S junctions taking into account the effect of opened tunnel channels on $I_{c}$, no definitive and quantitative comparison to theory can be made. However, since we expect approximately equal tunnel barriers at the $S$-2DEG interfaces and we found $T_{d} \leqslant \Gamma$, opened tunnel channels are expected to be present.

As was indicated above, extreme filtering of all measurement leads is essential to observe the correct low- 
temperature transport characteristics of our devices. The first filter is a $\Pi$ filter, mounted at room temperature, which filters rf signals. Next and more important is a cryogenic filter connected to the mixing chamber of the cryostat, which consists of a multipole $R C$ section and a $\mathrm{Cu}$-powder filter in series. It has a high-frequency attenuation better than $180 \mathrm{~dB}$. If this cryogenic filter is not included, transport measurements give fundamentally different results that cannot be modeled simply by an increased effective temperature. The $I_{c}(T)$ characteristic presented in Fig. 3 is obtained without cryogenic filters and does not show an exponential temperature dependence but instead saturates at $300 \mathrm{mK}$. The influence of external noise can also be seen by comparing $I-V$ curves of the 4- $\mu \mathrm{m}$ sample measured with and without cryogenic filtering. In Fig. 1 the hysteretic $I-V$ obtained with filtering is shown, which can be fitted reasonably well to the theoretical prediction for RSJ-type junctions. ${ }^{22}$ Without cryogenic filtering all hysteresis is suppressed, the critical current is reduced, and the curve has a finite slope for all bias currents as can be seen in the inset of Fig. 3 .

In summary, transport experiments on $S$-2DEG- $S$ junctions in the long and ballistic regime give seemingly incompatible results. From measurements of the resistance at finite bias and the critical current density it is clear that both nor- mal and Andreev reflection occur. However, the temperature dependence of the critical current suggests that it is carried by a few transport channels that do not suffer normal reflection. The disorder-induced opening of tunnel channels theory can be used to qualitatively explain these results.

Because the number of transport modes in $S$-2DEG- $S$ junctions can be tuned by changing their width and electron density they are a promising experimental system for a quantitative study of the opened channels. A rough estimate based on experimental parameters indicates that in the $0.5-\mu \mathrm{m}$ junction the number of open modes has already decreased to 1. A detailed analysis will require a theoretical description that gives information on the expected number of opened channels, the current they can carry, and the conditions required for opened channels to appear in these junctions. This theory might also explain the increase of $L^{*}$ as $W$ decreases.

We have benefited from discussions with Yuli Nazarov and Jeroen Wildöer. This research was financially supported by the Dutch Foundation for Fundamental Research on Matter (Stichting F.O.M.). The collaboration between the DUT and the EPFL is in the framework of the ESPRITQUANTEX project.
*Electronic address: luuk@sg.tn.tudelft.nl

${ }^{1}$ For a review see Vol. 203 of NATO Advanced Study Institute, Series B: Physics, edited by F.W.J. Hekking, G. Schön, and D.V. Averin (Plenum, New York, 1994).

${ }^{2}$ V.Z. Kresin, Phys. Rev. B 34, 7587 (1986).

${ }^{3}$ U. Schüssler and R. Kümmel, Phys. Rev. B 47, 2754 (1993).

${ }^{4}$ A. Chrestin, T. Matsuyama, and U. Merkt, Phys. Rev. B 49, 1391 (1994).

${ }^{5}$ C. Nguyen, H. Kroemer, and E.L. Hu, Phys. Rev. Lett. 69, 2847 (1992).

${ }^{6}$ B.J. van Wees et al., Physica B 203, 285 (1994).

${ }^{7}$ H. Takayanagi and T. Akazaki, Solid State Commun. 96 (11), 815 (1995).

${ }^{8}$ C.W.J. Beenakker, B. Rejaei, and J.A. Melsen, Phys. Rev. Lett. 72, 2470 (1994); Yu.V. Nazarov, ibid. 73, 134 (1994); J.A. Melsen and C.W.J. Beenakker, Physica (Amsterdam) 203B, 219 (1994)

${ }^{9}$ J.F. Carlin, A. Rudra, and M. Ilegems (unpublished).

${ }^{10}$ L.C. Mur et al. (unpublished).
${ }^{11}$ K. Kajiyama, Y. Mizushima, and S. Sakata, Appl. Phys. Lett. 23, 458 (1973).

${ }^{12}$ P.H.C. Magnée et al., Appl. Phys. Lett. 67, 3569 (1995).

${ }^{13}$ G.E. Blonder, M. Tinkham, and T.M. Klapwijk, Phys. Rev. B 25, 4515 (1982).

${ }^{14}$ R. Landauer, Philos. Mag. 21, 863 (1970).

${ }^{15}$ P.H.C. Magnée et al. Phys. Rev. B 52, R11 630 (1995).

${ }^{16}$ We use $I_{c} \propto \Gamma^{2}$ (Ref. 3) but do not know how to estimate the influence of $T_{d}$ on $I_{c}$.

${ }^{17}$ U. Gunsenheimer, U. Schüssler, and R. Kümmel, Phys. Rev. B 49, 6111 (1994).

${ }^{18}$ I.O. Kulik, Zh. Éksp. Teor. Fiz. 57, 1745 (1969) [Sov. Phys. JETP 30, 944 (1970)]; C. Ishii, Prog. Theor. Phys. 44, 1525 (1970); J. Bardeen and J.L. Johnson, Phys. Rev. B 5, 72 (1972).

${ }^{19}$ C.W.J. Beenakker, Phys. Rev. B 46, 12841 (1992).

${ }^{20}$ A. Kastalsky et al., Phys. Rev. Lett. 67, 3026 (1991).

${ }^{21}$ B.J. van Wees et al., Phys. Rev. Lett. 69, 510 (1992).

${ }^{22}$ T.P. Orlando and K.A. Delin, Foundations of Applied Superconductivity, 1st ed. (Addison-Wesley, New York, 1991). 